\title{
A CONSTRAINT ON THE MAXIMUM REFLECTANCE OF RAPIDLY OSCILLATING DIELECTRIC GRATINGS*
}

\author{
GANG $\mathrm{BAO}^{\dagger}$, DAVID C. DOBSON ${ }^{\ddagger}$, AND KARIM RAMDANI ${ }^{\dagger}$
}

\begin{abstract}
When considering optimal design problems involving diffraction gratings, it is useful to have some a priori characterization of the range of possible reflectances one can achieve for given material parameters. Here we consider the limiting case of a rapidly oscillating dielectric grating and show that such gratings can have reflectance no greater than that of a flat interface, regardless of the shape of the grating interface.
\end{abstract}

Key words. diffraction grating, optimal design, maximum reflectance

AMS subject classifications. 78A45, 93B03

PII. S036301290037435X

1. Introduction. A diffraction grating is formed by a periodic interface separating two homogeneous materials. In practical applications, one wishes to design the shape of the interface so that time-harmonic waves incident on the interface have a desired reflection and transmission pattern. Such design problems can be solved, for example, by optimization techniques [7] and homogenization [2]. An important question arising in this context is as follows: Given a particular class of admissible designs (interface shapes), which reflection and transmission patterns are attainable? In this paper we provide an answer in the case of blazed gratings (i.e., interfaces which can be represented by the graph of a function), which are rapidly oscillating with respect to the incident wavelength. The gratings are required to be dielectric. The basic result is a constraint on the reflectance, which says that in the limit as the grating period goes to zero the grating reflectance can be no greater than the reflectance obtained for a flat interface. This constraint holds regardless of the depth of the grating and the shape of the interface.

While rapidly oscillating gratings may seem to be of limited practical interest, they are, in fact, widely used. Optical engineers have been aware of homogenization effects in gratings for many years and often use high spatial frequency gratings to approximate corresponding multilayered structures (and vice-versa) [12]. The primary practical advantage of this approach is that material "layers" with intermediate refractive indices can be approximated by a grating composed of only two materials. In this way, the use of expensive, unstable, or nonexistent materials can often

* Received by the editors June 22, 2000; accepted for publication (in revised form) September 6, 2001; published electronically March 5, 2002. The U.S. Government is authorized to reproduce and distribute reprints for Governmental purposes notwithstanding any copyright notation thereon. The views and conclusions contained herein are those of the authors and should not be interpreted as necessarily representing the official policies or endorsements, either expressed or implied, of the Air Force Office of Scientific Research or the U.S. Government.

http://www.siam.org/journals/sicon/40-6/37435.html

${ }^{\dagger}$ Department of Mathematics, Michigan State University, East-Lansing, MI 48824 (bao@math. msu.edu, ramdani@math.msu.edu). The research of the first author was partially supported by the NSF Applied Mathematics Programs grant DMS 98-03604 (99-96416), the NSF University-Industry Cooperative Research Programs grants DMS 98-03809 and DMS 99-72292, the NSF Western Europe Programs grant INT 98-15798, and the Office of Naval Research (ONR) grant N000140010299.

${ }^{\ddagger}$ Department of Mathematics, Texas A\&M University, College Station, TX 77843-3368 (dobson@ math.tamu.edu). The research of this author was supported by the Air Force Office of Scientific Research, Air Force Material Command, USAF, under grant F49620-98-1-0005. The research of this author was also supported by NSF grant DMS 0072439 and an Alfred P. Sloan Research Fellowship. 
be avoided. One of the primary uses for rapidly oscillating gratings is in so-called moth-eye antireflective structures (see, e.g., $[1,8]$ and references therein), which are widely used to reduce glare on display devices and are commercially available.

Any optical engineer engaged in designing or optimizing a rapidly oscillating grating is faced with the question of whether or not a desired reflectance profile is attainable with given materials. This paper is aimed exactly at that question, showing that high reflectivity designs are generally not attainable with simple blazed high spatial frequency gratings. We consider the approach taken here as a first step toward solving the more difficult problem of characterizing attainable reflection/transmission patterns in more general diffraction gratings.

The plan of the paper is as follows. In the next section, we begin by analyzing the case of reflection from a "layered medium," i.e., a medium which has spatial dependence in only one direction. Under the condition that the refractive index of the medium is monotone in that direction, we establish the desired reflectance constraint. We conclude in section 3 by using homogenization theory to reduce the limiting case of a rapidly oscillating grating to the monotone layered medium. We prove that in the limit as the grating period goes to zero, the reflectance constraint is satisfied.

2. Layered medium case. We first consider a layered medium in $\mathbb{R}^{2}$, characterized by the real dielectric coefficient $k\left(x_{2}\right)$, where $x=\left(x_{1}, x_{2}\right) \in \mathbb{R}^{2}$. It is assumed that $k\left(x_{2}\right) \equiv k_{a}$ for $x_{2} \geq 0$ (i.e., in the "air"), and $k\left(x_{2}\right) \equiv k_{s}$ for $x_{2} \leq-b$, (i.e., in the "substrate"), where $0<b<\infty$ is an arbitrary depth. Consider an incoming plane wave $u_{i}=e^{i \alpha x_{1}+i \beta_{a} x_{2}}$, where

$$
\alpha=k_{a} \sin \theta, \quad \beta_{a}=k_{a} \cos \theta,
$$

and $|\theta|<\pi / 2$ is the angle of incidence with respect to the $x_{2}$-axis. We wish to find solutions $w$ satisfying the Helmholtz equation $\triangle w+k^{2} w=0$ in $\mathbb{R}^{2}$, plus appropriate outgoing wave conditions.

To make the problem independent of $x_{1}$, one can consider the functions $u=$ $w e^{-i \alpha x_{1}}$. Defining $\beta\left(x_{2}\right)=\sqrt{k\left(x_{2}\right)^{2}-\alpha^{2}}$ and setting $\beta_{s}=\beta(-b)$, we specify the reflection and transmission conditions

$$
\begin{array}{ll}
u\left(x_{2}\right)=e^{i \beta_{a} x_{2}}+r e^{-i \beta_{a} x_{2}} & \text { for } x_{2} \geq 0, \\
u\left(x_{2}\right)=t e^{i \beta_{s} x_{2}} & \text { for } x_{2} \leq-b,
\end{array}
$$

where the coefficients $r$ and $t$ are to be determined. This leads to the following boundary value problem in $x_{2}$ :

$$
\begin{aligned}
u^{\prime \prime}+\beta^{2} u & =0 \quad \text { in }(0,-b), \\
u^{\prime}(0) & =-i \beta_{a} u(0)+2 i \beta_{a}, \\
u^{\prime}(-b) & =i \beta_{s} u(-b) .
\end{aligned}
$$

In weak form, we have

$$
\int_{-b}^{0} u^{\prime} \bar{v}^{\prime}-\int_{-b}^{0} \beta^{2} u \bar{v}+i \beta_{a} u(0) \bar{v}(0)+i \beta_{s} u(-b) \bar{v}(-b)=2 i \beta_{a} \bar{v}(0) .
$$

Lemma 2.1. Let $\beta \in L^{\infty}(-b, 0)$ be real-valued. Then problem (3)-(5) admits a unique weak solution $u \in H^{1}(-b, 0)$.

Proof. We seek $u \in H^{1}(-b, 0)$ such that (6) is satisfied for all $v \in H^{1}(-b, 0)$. It is easy to rewrite this problem as a linear operator equation $u-A u=f$, where $A$ 
is compact (see, e.g., [7]). Applying the Fredholm alternative, existence then follows from uniqueness for the homogeneous problem $w-A w=0$.

Thus it suffices to prove uniqueness for the homogeneous problem

$$
\begin{aligned}
w^{\prime \prime}+\beta^{2} w & =0 \quad \text { in }(0,-b), \\
w^{\prime}(0) & =-i \beta_{a} w(0), \\
w^{\prime}(-b) & =i \beta_{s} w(-b),
\end{aligned}
$$

with associated weak form

$$
\int_{-b}^{0} w^{\prime} \bar{v}^{\prime}-\int_{-b}^{0} \beta^{2} w \bar{v}+i \beta_{a} w(0) \bar{v}(0)+i \beta_{s} w(-b) \bar{v}(-b)=0 .
$$

Note that any solution $w \in H^{1}(-b, 0)$ of $(10)$ is also in $H^{2}(-b, 0)$ since $w^{\prime \prime}=-\beta^{2} w$ a.e., and the right-hand side is in $L^{2}$. By Sobolev imbedding, $w \in C^{1}$. Setting $v=w$ in (10) and taking the imaginary part, we find that $w(-b)=w(0)=0$. From (8), (9) we also have $w^{\prime}(-b)=w^{\prime}(0)=0$. Uniqueness now follows by classical results for the Cauchy problem (see Hörmander [9, section 8.9] or Nirenberg [10]).

We can now investigate the properties of the reflectance of a given structure defined by $\beta\left(x_{2}\right)$. First, given the solution $u$ to (3)-(5), we define the reflection coefficient $r=u(0)-1$, and the reflectance $\mathcal{R}=|r|^{2}$. The reflectance represents the proportion of incident energy reflected from the structure. Similarly, we define the transmission coefficient $t=u(-b)$, and the transmittance $\mathcal{T}=\left(\beta_{s} / \beta_{a}\right)|t|^{2}$. Setting $v=u$ and taking the imaginary part of the resulting equality in (6) yield conservation of energy:

$$
\mathcal{R}+\mathcal{T}=1
$$

Now taking $v=u^{\prime}$ and applying the identities (3)-(5), one finds from (6) that

$$
\beta_{s}^{2}|u(-b)|^{2}-\beta_{a}^{2}\left\{|u(0)|^{2}-4 \operatorname{Re} u(0)-4\right\}=\int_{-b}^{0} \beta^{2}\left(u^{\prime} \bar{u}+u \bar{u}^{\prime}\right) .
$$

Integrating the last term in (12) by parts, we have

$$
\int_{-b}^{0} \beta^{2}\left(u^{\prime} \bar{u}+u \bar{u}^{\prime}\right)=-\int_{-b}^{0}\left(\beta^{2}\right)^{\prime}|u|^{2}+\beta_{a}^{2}|u(0)|^{2}-\beta_{s}^{2}|u(-b)|^{2} .
$$

Then (12) becomes

$$
2 \beta_{s}^{2}|u(-b)|^{2}-2 \beta_{a}^{2}|u(0)-1|^{2}-2 \beta_{a}^{2}=-\int\left(\beta^{2}\right)^{\prime}|u|^{2} .
$$

Applying conservation of energy (11), $|t|^{2}=\left(\beta_{a} / \beta_{s}\right)\left(1-|r|^{2}\right)$ so that (13) yields

$$
\mathcal{R}=\frac{\beta_{s}-\beta_{a}}{\beta_{s}+\beta_{a}}+\frac{1}{2 \beta_{a}\left(\beta_{a}+\beta_{s}\right)} \int_{-b}^{0}\left(\beta^{2}\right)^{\prime}|u|^{2} .
$$

Since $\beta^{2}$ is nonincreasing, we immediately obtain that

$$
\mathcal{R} \leq \frac{\beta_{s}-\beta_{a}}{\beta_{s}+\beta_{a}} .
$$


The term on the right is the square root of the reflectance in the case of a flat profile (see, e.g., Born and Wolf [4] for a complete discussion of reflectance from flat interfaces). To improve this estimate, we need a lower bound on $|u|^{2}$.

Lemma 2.2. Suppose $\beta\left(x_{2}\right)$ is nonincreasing. Then the solution $u$ of (3)-(5) satisfies

$$
|u|^{2} \geq|t|^{2}
$$

where $t=u(-b)$ is the transmission coefficient.

Proof. First suppose that $k\left(x_{2}\right)$ is composed of a finite number of homogeneous layers, with refractive indices $k_{a} \leq k_{1} \leq k_{2} \leq \cdots \leq k_{n} \leq k_{s}$, with depths $h_{1}, \ldots, h_{n}$; i.e., setting $b_{j}=\sum_{k=1}^{j} h_{k}$, we have $k\left(x_{2}\right)=k_{j}$ for $-b_{j} \leq x_{2} \leq-b_{j-1}$. Set $b=b_{n}$.

Letting $u(-b)=t$, the boundary condition (5) is $u^{\prime}(-b)=i \beta_{s} t$. Solving for $u$ in the $n$th layer, $-b_{n} \leq x \leq-b_{n-1}$, one obtains

$$
u(x)=t\left(\cos \beta_{n}\left(x+b_{n}\right)+i\left(\beta_{s} / \beta_{n}\right) \sin \beta_{n}\left(x+b_{n}\right)\right) e^{-i \beta_{s} b_{n}} .
$$

Note that since $\beta_{s} / \beta_{n} \geq 1$, we have $|u(x)|^{2} \geq|t|^{2}$. Having obtained $u$ in terms of $t$ the $n$th layer, one can now continue propagating the solution upward layer by layer, each time obtaining a solution in the form

$$
u(x)=\tilde{t}_{j}\left(q_{1} \cos \theta+i q_{2}\left(\beta_{j} / \beta_{j-1}\right) \sin \theta\right),
$$

where $\tilde{t}_{j}$ is a complex constant with $\left|\tilde{t}_{j}\right|^{2} \geq|t|^{2}$, and $q_{1}$ and $q_{2}$ are complex constants in the form

$$
\begin{aligned}
& q_{1}=\cos \phi+i\left(\beta_{j+1} / \beta_{j}\right) \sin \phi \\
& q_{2}=i \sin \phi+\left(\beta_{j+1} / \beta_{j}\right) \cos \phi
\end{aligned}
$$

The result follows from the fact that the complex number $Z=q_{1} \cos \theta+i q_{2}\left(\beta_{j}\right)$ $\left.\beta_{j-1}\right) \sin \theta$ satisfies $|Z| \geq 1$. Indeed, setting $\gamma_{j}=\beta_{j} / \beta_{j-1}$, we have

$$
\begin{aligned}
|Z|^{2}= & \left(\cos \theta \cos \phi-\gamma_{j} \sin \theta \sin \phi\right)^{2}+\gamma_{j+1}^{2}\left(\cos \theta \sin \phi+\gamma_{j} \sin \theta \cos \phi\right)^{2} \\
= & \cos ^{2} \theta\left(\cos ^{2} \phi+\gamma_{j+1}^{2} \sin ^{2} \phi\right)+\gamma_{j}^{2} \sin ^{2} \theta\left(\sin ^{2} \phi+\gamma_{j+1}^{2} \cos ^{2} \phi\right) \\
& +2 \gamma_{j}\left(\gamma_{j+1}^{2}-1\right) \cos \theta \cos \phi \sin \theta \sin \phi \\
= & \cos ^{2} \theta\left(1+\left(\gamma_{j+1}^{2}-1\right) \sin ^{2} \phi\right)+\gamma_{j}^{2} \sin ^{2} \theta\left(1+\left(\gamma_{j+1}^{2}-1\right) \cos ^{2} \phi\right) \\
& +2 \gamma_{j}\left(\gamma_{j+1}^{2}-1\right) \cos \theta \cos \phi \sin \theta \sin \phi .
\end{aligned}
$$

Thus $|Z|^{2}$ can be written as

$$
|Z|^{2}=\cos ^{2} \theta+\gamma_{j}^{2} \sin ^{2} \theta+\left(\gamma_{j+1}^{2}-1\right)\left(\cos \theta \sin \phi+\gamma_{j} \sin \theta \cos \phi\right)^{2} .
$$

Since $\beta$ is a nonincreasing function, we have $\gamma_{j}=\beta_{j} / \beta_{j-1} \geq 1$, and thus $|Z| \geq 1$. Consequently, $|u(x)| \geq|t|^{2}$ in each layer.

In a manner exactly analogous to the procedure above, one can also obtain the estimate

$$
|u(x)|^{2} \leq|u(0)|^{2}+|2-u(0)|^{2} \quad \text { for } x \leq 0
$$


Since $|u(0)-1|^{2}=\mathcal{R} \leq 1$, it follows that $\int_{-b}^{0}|u|^{2} \leq C$, where $C$ is independent of the piecewise constant function $\beta$, provided only that $\beta$ is nonincreasing. Taking the real part of the bilinear form (6) with $v=u$, we then find immediately that

$$
\|u\|_{H^{1}} \leq C,
$$

where $C$ now depends only on $b, \beta_{a}, \beta_{s}$.

The general case of nonincreasing $\beta \in L^{\infty}$ is now handled easily by approximation. Specifically, let $\left\{\beta^{k}\right\}$ be a sequence of nondecreasing, piecewise constant functions converging to a given $\beta$ in the weak $* L^{\infty}$ sense. Let $u_{k}$ be the corresponding sequence of solutions to (3)-(5). By (16), $\left\|u_{k}\right\|_{H^{1}} \leq C$; hence there exists a subsequence (still denoted $u_{k}$ ) converging weakly in $H^{1}$ and strongly in $L^{2}$ to some $\tilde{u}$. It follows that, for every fixed $v \in H^{1}$,

$$
\begin{array}{r}
2 i \beta_{a} \bar{v}(0)=\int_{-b}^{0} u_{k}^{\prime} \bar{v}^{\prime}-\int_{-b}^{0}\left(\beta^{k}\right)^{2} u_{k} \bar{v}+i \beta_{a} u_{k}(0) \bar{v}(0)+i \beta_{s} u_{k}(-b) \bar{v}(-b) \\
\rightarrow \int_{-b}^{0} \tilde{u}^{\prime} \bar{v}^{\prime}-\int_{-b}^{0} \beta^{2} \tilde{u} \bar{v}+i \beta_{a} \tilde{u}(0) \bar{v}(0)+i \beta_{s} \tilde{u}(-b) \bar{v}(-b)
\end{array}
$$

so that by Lemma $2.1, \tilde{u}=u$, the unique solution to (3)-(5). Finally, since $u_{k}$, $k=1,2, \ldots$, along with $u$ are uniformly bounded in $H^{2}$ and hence in $C^{1}$, we see that the convergence $u_{k} \rightarrow u$ is actually pointwise. Since the estimate $\left|u_{k}(x)\right|^{2} \geq\left|t_{k}\right|^{2}$ holds for each $k$, it must also hold for $u, t$.

Lemma 2.3. Suppose $\beta\left(x_{2}\right)$ is nonincreasing. Then

$$
\mathcal{R} \leq\left(\frac{\beta_{s}-\beta_{a}}{\beta_{s}+\beta_{a}}\right)^{2} .
$$

Proof. Using the previous lemma, we find that

$$
\int_{-b}^{0}\left(\beta^{2}\right)^{\prime}|u|^{2} \leq|t|^{2}\left(\beta_{a}^{2}-\beta_{s}^{2}\right)
$$

Noting that $|t|^{2}=\left(\beta_{a} / \beta_{s}\right)(1-\mathcal{R})$, the identity (14) then yields the desired estimate with a simple manipulation.

The estimate in Lemma 2.3 is sharp. Equality is attained for a sharp interface between two media with refractive indices $k_{a}$ and $k_{s}$. Thus the reflectance produced by any nonincreasing refractive index $k\left(x_{2}\right)$ with $k(-b)=k_{s}$ and $k(0)=k_{a}$ can be no more than the reflectance produced by the piecewise constant $k_{c}\left(x_{2}\right)$ with $k_{c}\left(x_{2}\right)=k_{s}$ for $x_{2}<a$ and $k_{c}\left(x_{2}\right)=k_{a}$ for $x_{2}>a, a \in(-b, 0)$. Incidentally, it is well known in engineering that for a fixed incidence angle one can create a layered structure with $\mathcal{R}$ lying anywhere in the interval $\left[0, R_{\max }\right]$, with $R_{\max }=\left(\left(\beta_{s}-\beta_{a}\right) /\left(\beta_{s}+\beta_{a}\right)\right)^{2}$. The key point here is that $\mathcal{R}$ cannot exceed $R_{\max }$ with nonincreasing $\beta$.

3. Rapidly oscillating case. We now consider the case of a rapidly oscillating dielectric grating. Specifically, suppose that we are given a grating structure with period $L$. By rescaling the problem, it suffices to consider the case $L=2 \pi$. Let $f \in L^{\infty}(\mathbb{R})$ be $2 \pi$-periodic; i.e., let

$$
f\left(x_{1}\right)=f\left(x_{1}+2 \pi n\right) \quad \text { a.e. in } x_{1}, \text { for all integers } n
$$


and satisfy

$$
-b<\inf f \leq \sup f<0 .
$$

The function $f$ represents an interface between two homogeneous materials with refractive indices $k_{a}$ and $k_{s}$. Define a corresponding refractive index function on $\mathbb{R}^{2}$ :

$$
\rho_{f}(x)= \begin{cases}k_{a}^{2} & \text { if } x_{2}>f\left(x_{1}\right), \\ k_{s}^{2} & \text { otherwise. }\end{cases}
$$

As in the previous section, given an incoming plane wave from above $u_{i}=$ $e^{i \alpha x_{1}+i \beta_{a}\left(x_{2}\right)}$ (where $\alpha$ and $\beta_{a}$ are as defined in (1)), we seek solutions of the Helmholtz equation $\triangle w+\rho_{f} w=0$, where $w$ is a sum of the incoming and scattered fields and satisfies appropriate outgoing wave conditions. The standard approach to solving this problem is to search for "quasi-periodic" solutions, that is, solutions $w$ such that $u=w e^{-i \alpha x_{1}}$ is $2 \pi$-periodic in $x_{1}$. A well-known procedure exists for formulating the problem variationally. This is outlined, for example, in $[2,7]$. The basic idea is to expand the periodic functions $u$ in a Fourier series in $x_{1}$ and match the solutions with the fundamental solution in the homogeneous regions $x_{2}>0$ and $x_{2}<-b$. This leads naturally to a Fourier series expansion for the Dirichlet-to-Neumann operators on the boundaries $\left\{x_{2}=0\right\}$ and $\left\{x_{2}=-b\right\}$. Defining the cylindrical domain $\Omega=(\mathbb{R} \times(-b, 0)) /(2 \pi Z \times\{0\})$ and the periodic boundaries $\Gamma_{a}$ corresponding to $\left\{x_{2}=0\right\}$ and $\Gamma_{s}$ corresponding to $\left\{x_{2}=-b\right\}$, the problem can then be formulated as

$$
\begin{array}{ll}
\triangle_{\alpha} u+\rho_{f} u=0 & \text { in } \Omega, \\
T_{a} u-\frac{\partial u}{\partial x_{2}}=2 i \beta_{a} & \text { on } \Gamma_{a}, \\
T_{s} u+\frac{\partial u}{\partial x_{2}}=0 & \text { on } \Gamma_{s},
\end{array}
$$

where $\triangle_{\alpha}=\triangle+2 i \alpha \partial_{1}-\alpha^{2}$. The Dirichlet-to-Neumann operators $T_{j}$ are defined by

$$
\left(T_{j} \phi\right)\left(x_{1}\right)=\sum_{n \in Z} i \beta_{j}^{n} \phi_{n} e^{i n x_{1}}, \quad j=a, s,
$$

where

$$
\beta_{j}^{n}= \begin{cases}\sqrt{k_{j}^{2}-(n+\alpha)^{2}} & \text { if } k_{j}^{2} \geq(n+\alpha)^{2}, \\ i \sqrt{k_{j}^{2}-(n+\alpha)^{2}} & \text { if } k_{j}^{2}<(n+\alpha)^{2},\end{cases}
$$

and $\phi_{n}$ denote the Fourier coefficients of $\phi$. To obtain the weak form, we define for $u, v \in H^{1}(\Omega)$

$$
B_{\rho_{f}}(u, v) \equiv \int_{\Omega}(\nabla+i \underline{\alpha}) u \cdot \overline{(\nabla+i \underline{\alpha}) v}-\int_{\Omega} \rho_{f} u \bar{v}-\int_{\Gamma_{a}}\left(T_{a} u\right) \bar{v}+\int_{\Gamma_{s}}\left(T_{s} u\right) \bar{v},
$$

where $\underline{\alpha}=(\alpha, 0)$ and

$$
g(v)=-2 i \beta_{a} \int_{\Gamma_{a}} \bar{v}
$$

We then wish to find $u \in H^{1}(\Omega)$ such that

$$
B_{\rho_{f}}(u, v)=g(v) \text { for all } v \in H^{1}(\Omega) .
$$


It is well known that a unique solution $u \in H^{1}(\Omega)$ of problem (20) exists for all but possibly a discrete set of parameters $k_{a}, k_{s}$ (see [3]). In addition, using a perturbation argument, the following lemma is proved in [6].

LEMma 3.1. Provided that the incoming wave is sufficiently low-frequency $\left(k_{a}, k_{s}\right.$ are sufficiently small), problem (20) admits a unique weak solution for all $f$ satisfying (18). Furthermore, the solutions $u$ are bounded in $H^{1}(\Omega)$ independently of $f$.

Remark. Under the conditions of Lemma 3.1, solutions are actually uniformly bounded in $H^{2}$, independent of $f$. This follows immediately from the equation

$$
\triangle u=-2 i \alpha \partial_{1} u+\left(\alpha^{2}-\rho_{f}\right) u .
$$

The $H^{1}$ bound on $u$ and the $L^{\infty}$ bound on $\rho_{f}$ guarantee that $\|\triangle u\|_{L^{2}} \leq C$, giving the $H^{2}$ estimate.

Once the solution to problem (20) has been determined, one can easily find the scattered far-field. The Rayleigh expansion [11] dictates that the field above $\left\{x_{2}=0\right\}$ must be in the form

$$
u\left(x_{1}, x_{2}\right)=\sum_{n=-\infty}^{\infty} r_{n} e^{i\left(n x_{1}-\beta_{a}^{n} x_{2}\right)},
$$

where the $r_{n}$ are unknown scalars. Matching this expansion with the boundary conditions for the variational solution, one finds that $r_{0}$, which corresponds to the "zero order" reflected mode, must be given by

$$
r_{0}=\frac{1}{2 \pi} \int_{0}^{2 \pi} u\left(x_{1}, 0\right) d x_{1}-1 .
$$

By rescaling the problem, one can see easily that for a sufficiently small grating period $L$ the coefficients $\beta_{a}^{n}$ are real only for $n=0$. This means that only the zero order mode propagates. Similarly, using the Rayleigh expansion in the region $x_{2}<-b$ and the fact that the grating period $L$ is small, one finds the lone transmitted mode $t_{0}=\frac{1}{2 \pi} \int_{0}^{2 \pi} u\left(x_{1},-b\right) d x_{1}$.

As in the layered medium case, setting the reflectance $\mathcal{R}_{0}=\left|r_{0}\right|^{2}$ and the transmittance $\mathcal{T}_{0}=\left|t_{0}\right|^{2}$, one can easily verify conservation of energy $\mathcal{R}_{0}+\mathcal{T}_{0}=1$ [7]. We would like to show that a reflectance bound similar to (17) holds in the grating case.

For $n=1,2, \ldots$, define $\rho_{n}\left(x_{1}, x_{2}\right)=\rho_{f}\left(n x_{1}, x_{2}\right)$. Thus $\rho_{n}$ represents a $2 \pi$ periodic grating oscillating more and more rapidly as $n$ increases. It is easily verified that $\rho_{n} \rightarrow \tilde{\rho}$ in the weak $* L^{\infty}(\Omega)$ sense, where

$$
\tilde{\rho}\left(x_{1}, x_{2}\right)=\frac{1}{2 \pi} \int_{0}^{2 \pi} \rho_{f}\left(x_{1}, x_{2}\right) d x_{1} .
$$

Note that $\tilde{\rho}$ is independent of $x_{1}$. Furthermore, due to the form of $\rho_{f}(19)$ and the fact that $k_{a}^{2} \leq k_{s}^{2}$, it is easy to see that $\tilde{\rho}$ is nonincreasing in $x_{2}$.

We can now state the main result of this paper.

THEOREM 3.2. Assume the conditions of Lemma 3.1. Given an arbitrary grating profile $f$ and any $\epsilon>0$, there exists a grating period $L$ such that when the profile $f$ is produced with period $L$ or less, the reflectance $\mathcal{R}_{0}$ resulting from $f$ satisfies

$$
\mathcal{R}_{0} \leq\left(\frac{\beta_{s}^{0}-\beta_{a}^{0}}{\beta_{s}^{0}+\beta_{a}^{0}}\right)^{2}+\epsilon .
$$


Thus, analogous to the layered medium case, for rapidly oscillating gratings the reflectance can be no more than the reflectance of a sharp interface between materials $k_{a}$ and $k_{b}$ plus a small error, regardless of the grating shape.

Proof. Since the bound (17) holds for $\tilde{\rho}$, inequality (22) is simply a statement of the continuity of $\mathcal{R}_{0}(\rho)$ with respect to weak $* L^{\infty}$ convergence $\rho_{n} \rightarrow \tilde{\rho}$. This is easy to prove. Let $u_{n}$ denote the sequence of solutions to problem (20) corresponding to the coefficients $\rho_{n}$. By Lemma 3.1, $\left\|u_{n}\right\|_{H^{1}}$ is uniformly bounded; hence each subsequence of $\left\{u_{n}\right\}$ has a further subsequence $\left\{u_{n^{\prime}}\right\}$ which converges weakly in $H^{1}$ to some $u \in H^{1}$. We show that the weak limit $u$ of each such subsequence is the same, thus proving that the original sequence $\left\{u_{n}\right\}$ converges weakly to $u$.

Holding $v \in H^{1}$ fixed, observe that

$$
\begin{aligned}
B_{\rho_{n^{\prime}}}(u, v)-B_{\rho_{n^{\prime}}}\left(u_{n^{\prime}}, v\right) & =\int_{\Omega}(\nabla+i \underline{\alpha})\left(u-u_{n^{\prime}}\right) \cdot \overline{(\nabla+i \underline{\alpha}) v} \\
& -\int_{\Omega} \rho_{n^{\prime}}\left(u-u_{n^{\prime}}\right) \bar{v}-\int_{\Gamma_{a}}\left(T_{a}\left(u-u_{n^{\prime}}\right)\right) \bar{v}+\int_{\Gamma_{s}}\left(T_{s}\left(u-u_{n^{\prime}}\right)\right) \bar{v} .
\end{aligned}
$$

Since $u_{n^{\prime}} \rightarrow u$ in $H^{1}$ and the operators $T_{j}$ are bounded maps from $H^{1 / 2}\left(\Gamma_{j}\right)$ into $H^{-1 / 2}\left(\Gamma_{j}\right)$, the first integral and the last two integrals vanish as $n^{\prime} \rightarrow \infty$. Further, the weak convergence of $u_{n^{\prime}}$ in $H^{1}$ implies strong convergence in $L^{2}$ so that

$$
\left|\int_{\Omega} \rho_{n^{\prime}}\left(u-u_{n^{\prime}}\right) \bar{v}\right| \leq\left\|\rho_{n^{\prime}}\right\|_{L^{\infty}}\left\|u-u_{n^{\prime}}\right\|_{L^{2}}\|v\|_{L^{2}} \rightarrow 0 .
$$

Thus $B_{\rho_{n^{\prime}}}(u, v) \rightarrow B_{\rho_{n^{\prime}}}\left(u_{n^{\prime}}, v\right)=g(v)$. The convergence $\rho_{n^{\prime}} \stackrel{*}{\rightarrow} \tilde{\rho}$ implies $B_{\rho_{n^{\prime}}}(u, v) \rightarrow$ $B_{\tilde{\rho}}(u, v)$. Hence $B_{\tilde{\rho}}(u, v)=g(v)$ for all $v$; i.e., $u$ solves $(20)$ for $\tilde{\rho}$. Since the solution $u$ is unique by Lemma 3.1, we conclude that the original sequence $u_{n} \rightarrow u$ weakly in $H^{1}$.

Since the traces $\left.\left.u_{n}\right|_{\Gamma_{a}} \rightarrow u\right|_{\Gamma_{a}}$ weakly in $H^{1 / 2}$, it follows by the definition (21) that the corresponding reflection coefficients, and hence the reflectances, converge.

Acknowledgments. The authors wish to thank Jeff Rauch for raising the question which led to this work. They are also very grateful to the referees and the associate editor for their helpful comments, which improved the paper greatly.

\section{REFERENCES}

[1] M. Auslender, D. Levy, AND S. Hava, Design and analysis of antireflection grating structure for solar energy absorber, in Optical Materials Technology for Energy Efficiency and Solar Energy Conversion XV, C.M. Lampert, C.-G. Granqvist, M. Graetzel, and S. Deb, eds., Proc. SPIE 3138, SPIE, Bellingham, WA, 1997, pp. 160-165.

[2] G. Bao And E. Bonnetier, Optimal design of periodic diffractive structures in TM polarization, Appl. Math. Optim., 43 (2001), pp. 103-116.

[3] G. BaO, D. C. Dobson, And J. A. Cox, Mathematical studies of rigorous grating theory, J. Opt. Soc. Amer. A, 12 (1995), pp. 1029-1042.

[4] M. Born And E. Wolf, Principles of Optics, Electromagnetic Theory of Propagation Interference and Diffraction of Light, 6th ed., Pergamon Press, Oxford, UK, 1980.

[5] P. G. Ciarlet, The Finite Element Method for Elliptic Problems, North-Holland, Amsterdam, 1978.

[6] D. C. Dobson, Optimal design of periodic antireflective structures for the Helmholtz equation, European. J. Appl. Math., 4 (1993), pp. 321-340.

[7] D. C. Dobson, Optimal shape design of blazed diffraction gratings, Appl. Math. Optim., 40 (1999), pp. 61-78. 
[8] A. B. Harker and J. F. Denatale, Diamond gradient index "moth-eye" antireflection surfaces for LWIR windows, in Window and Dome Technologies and Materials III, Paul Klocek, ed., Proc. SPIE 1760, Bellingham, WA, 1992, pp. 261-267.

[9] L. Hörmander, Linear Partial Differential Operators, Springer-Verlag, Berlin, 1969.

[10] L. Nirenberg, Uniqueness in Cauchy problems for differential operators with constant leading coefficients, Comm. Pure Appl. Math., 10 (1957), pp. 89-105.

[11] R. Petit, ED., Electromagnetic Theory of Gratings, Topics in Current Physics 22, SpringerVerlag, Berlin, New York, 1980.

[12] R. Petit And G. Bouchitté, Replacement of a very fine grating by a stratified layer: Homogenization techniques, and the multiple scale method, in Proceedings of the International Conference on the Application and Theory of Periodic Structures, Diffraction Gratings, and Moire Phenomena III, Jeremy M. Lerner, ed., Proc. SPIE 815, Bellingham, WA, 1988, pp. $25-31$. 